\title{
Thermal and utility properties of amide clay modifying agents
}

\author{
Krzysztof Garman • Ewa Olewnik • \\ Wojciech Czerwiński
}

CEEC-TAC1 Conference Special Issue

(C) The Author(s) 2012. This article is published with open access at Springerlink.com

\begin{abstract}
This article presents the results of investigation into receiving amide modification agents of smectic clays, used as nanofillers in polymer nanocomposites. Prepared materials were obtained on the base of terephthalic and isophthalic acids and simple aliphatic amines. Such structure makes them good layered silicates modifying agents as well as makes them well affiliate to structure of polymers containing aromatic, carbonyl, amide, etc. groups. Obtained compounds were introduced into clays' structure in protonated form, according to formulated method. Conducted research confirmed that the modification of montmorillonite clay (MMT) with received compounds had taken place. In order to establish that fact elementary analysis and X-ray diffraction (XRD) methods were used. Modificator molecules must display thermal resistance in full range of polymer processing temperatures. To investigate these properties the Thermogravimetric analysis (TG) of obtained compounds was carried out. These studies indicate that among the obtained compounds there were the ones with thermal stability over $523 \mathrm{~K}$. Thermal resistance makes it possible for these substances to be used in poly(ethylene terephthalate) processing.
\end{abstract}

Keywords Nanocomposites - Clay modifiers - Amide · Montmorillonite - Thermal properties .

Poly(ethylene terephthalate)

K. Garman $(\bowtie) \cdot$ E. Olewnik · W. Czerwiński

Faculty of Chemistry, Nicolaus Copernicus University,

7 Gagarin Street, 87-100 Toruń, Poland

e-mail: kgarman@chem.uni.torun.pl

\section{Introduction}

Polymer nanocomposites have got several properties (e.g., an increased flame retardancy) that make them more appealing to introduce in commonly used products than non-composite polymer materials. A reinforcement of structure of polymer nanocomposites has also been observed which results in the improvement of their mechanical properties like hardness, ultimate tensile stress or general durability. It is known that polymer nanocomposites display electroconductive and barrier properties. The development of possible applications of polymer nanocomposites is not only correlated with their durability, barrier, and flame-retardant properties but also with their biocompatibility $[1,2]$. Polymer nanocomposites are obtained by introduction of, e.g., either small molecules like silicas, silica, or titania oxides or macromolecules like whisker-structure cellulose fibers or carbon nanotubes into the polymer matrix in an as well-dispersed way as possible [3-5].

Aiming to obtain polymer nanocomposites, on the base of hydrophobic polymer and naturally hydrophilic MMT clay (most popular silicate polymer filler), it is necessary to make them compatible [6]. The most common method is to modify the surface of the clay with specific modifying agents. These agents need to be amphiphilic and suitable to be introduced into the clay in the cationic form, thanks to the high cation-exchange capacity of the MMT.

There are well-known aliphatic clay modifiers (the most common are, e.g., protonated primary alkyloamines, quaternary alkylammonium salts, quaternary phosphonnium salts), applied in the process of obtaining polyolefin composites, but there is still lack of publicized researched concerning aromatic modifiers [7]. Molecules containing 
aromatic groups in their structure becomes clay modifying agents and also make them moleculary compatible to selected polymers, e.g., poly(ethylene terephthalate).

\section{Materials}

Terephthaloil chloride (p.p.a., Aldrich, Germany), 5-aminoizophthalic acid (p.p.a., Acros Organics, Belgium), ethanolamine (p.p.a., Chempur, Poland), diethylamine (p.p.a., Chempur, Poland), butylamine (p.p.a., Aldrich, Germany), dibutylamine (p.p.a., Aldrich, Germany), chloroform (p.p.a., POCH, Poland), dimethyl sulfoxide (p.p.a., Scharlan Chemie, Spain) were used in the carried out synthesis.

Modifying agents were used to organophilise smectic clay Bentonite clay K-10 (Acros Organics, Belgium).

\section{Experimental}

The series of compounds (shown at Fig. 1): $N, N^{\prime}$-bis-(2hydroxy-ethyl)-terephthalamide (denoted as MET), $N, N^{\prime}$ dibutyl-terephthalamide (denoted as MBT), $N, N, N^{\prime}, N^{\prime}$-tetraethyl-terephthalamide (denoted as DET), $N, N, N^{\prime}, N^{\prime}$-tetrabutyl-terephthalamide (denoted as DBT), 5-amino- $N, N^{\prime}$ bis-(2-hydroxy-ethyl)-isophthalamide (denoted as MEI), 5-amino- $N, N, N^{\prime}, N^{\prime}$-tetraethyl-isophthalamide (denoted as DEI), 5-amino- $N, N, N^{\prime}, N^{\prime}$-tetrabutyl-isophthalamide (denoted as DBI) were synthesized and then used as MMT modifiers. The methods of synthesis and modification procedures are presented below.
Synthesis

On the bases of already known and described methods of receiving amides, a new method of amide-derivatives of terephthalic and 5-amino-isophthalic acids was accomplished [8-11]. Syntheses were carried out in two-phase system, where proper amine was added into the chloroform solution of terephthaloil chloride. Reaction was quenched by adding water. Afterwards phases were separated, solvent was evaporated and obtained product was dried for $24 \mathrm{~h}$ at $353 \mathrm{~K}$ temperature. In the case of 5-amine-isophthaloilamides, acid was dissolved in dimethyl sulfoxide and consequently proper amine was dropped into the intensively stirred solution. Precipitated pink (MEI) and white (DEI and DBI) products were filtered, washed with solvent and dried for $24 \mathrm{~h}$ at $353 \mathrm{~K}$ temperature.

\section{Clay modification}

Modification of MMT clay was conducted directly with previously evaluated procedure [12]. The amount of modifier was added into the previously prepared water-methanol solution. Proportions of ingredients varied depending on the modifier used. While continuously stirring, the mixture was acidified with hyrdochloric acid in molar ratio of $\mathrm{HCl}$ to proton-acceptor nitrogen atoms of modifier like 2:1. The system was heated up to $349 \mathrm{~K}$ and MMT clay was added. The ratio of modifier to clay quantity was set as $2 \mathrm{mmol}$ of modifier per $1 \mathrm{~g}$ of the clay. The modification process was carried out at $349 \mathrm{~K}$ for $3.5 \mathrm{~h}$. The mixture was filtered, washed with water-methanol solution (composition

Fig. 1 Synthesized amidesMMT modifying agents

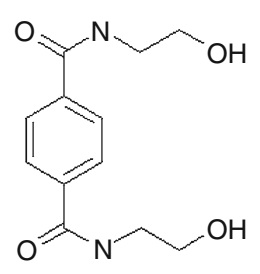

MET

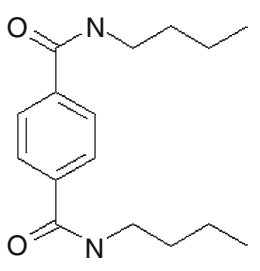

MBT<smiles>CCN(CC)C(=O)c1ccc(C(=O)N(CC)CC)cc1</smiles>

DET<smiles>CCCCN(CCCC)C(=O)c1ccc(C(=O)N(CCCC)CCCC)cc1</smiles>

DBT<smiles>CCCNC(=O)c1cc(N)cc(C(=O)NCCO)c1</smiles><smiles>CCN(CC)C(=O)c1cc(N)cc(C(=O)N(CC)CC)c1</smiles><smiles>CCCCN(CCCC)C(=O)c1cc(N)cc(C(=O)N(CCCC)CCCC)c1</smiles> 
adequate to modifying mixture solution) until no chloride ions were observed in test with the drop of silver(I) nitrate solution. Modified clays were dried at $353 \mathrm{~K}$ for $24 \mathrm{~h}$.

Modification of MMT with MET, MBT, DET, DBT, MEI, DEI, DBI lead to obtain products denoted as MMTMET, MMT-MBT, MMT-DET, MMT-DBT, MMT-MEI, MMT-DEI, MMT-DBI, respectively.

Experimental procedures

Synthesized modifiers were verified by means of the nuclear magnetic resonance (NMR) analysis, using Bruker $300 \mathrm{MHz}$ spectrometer.

The modification processes were determined with the XRD analyses to describe the changes of clays' intergallery distance. The analyses were carried out by means of the Philips X-PERT PRO diffractometer with the parameters: angle range $1-12^{\circ} 2 \theta$, divergence slit $0.125^{\circ}$, receiving slit $0.5^{\circ}$, anti-scatter slit $0.25^{\circ}$, step $0.017^{\circ}$, and step time $10 \mathrm{~s}$.

Elementary analysis was conducted in order to determine the amount of modifiers effectively used in particular processes. Analyses were carried out by means of the Vario MACRO CHN Elementary Analyzer form Analysensysteme GmbH (Germany).

Thermal properties of all products were determined using Thermal Analysis (by means of TA Instruments, SDT 2960 Simultaneous DSC-TGA) with heating rate $10 \mathrm{~K} \mathrm{~min}^{-1}$ in range $295-873 \mathrm{~K}$ with air flow over samples. The mass of the samples was 3.2-10.8 mg. During the analysis, the alumina crucibles were used.

\section{Results and discussion}

Thermogravimetric analysis

Thermal properties of all products were determined with thermal analysis methods. In this article, results of thermogravimetric analysis (TG) are shown to describe mainly temperatures of thermal decomposition. It is done due to main requirements of polymer fillers-thermal stability of all components in polymer processing temperatures.

In Table 1, the temperatures of decomposition of investigated modifiers are shown. MET displays the greatest thermal resistance, decomposing at $527 \mathrm{~K}$. So it is possible to apply MET as modifying agent of smectic clays used as poly(ethylene terephthalate) nanofillers. Moreover, Fig. 2 presents the collective thermogram of modifiers prepared on the basis of terephthalic acid. The collective thermogram of modifiers based on 5-aminoisophthalic acid is presented in Fig. 3. Thermograms were collected in temperature range $298-873 \mathrm{~K}$ with heating rate $10 \mathrm{~K} \mathrm{~min}^{-1}$ in the atmosphere of air. In addition, the usage of air during
Table 1 The thermal stability of modifiers

\begin{tabular}{ll}
\hline Modifier & $\begin{array}{l}\text { Temperature of } \\
\text { decomposition/K }\end{array}$ \\
\hline MET & 527 \\
MBT & 496 \\
DET & 439 \\
DBT & 458 \\
MEI & 463 \\
DEI & 461 \\
DBI & 478 \\
\hline
\end{tabular}

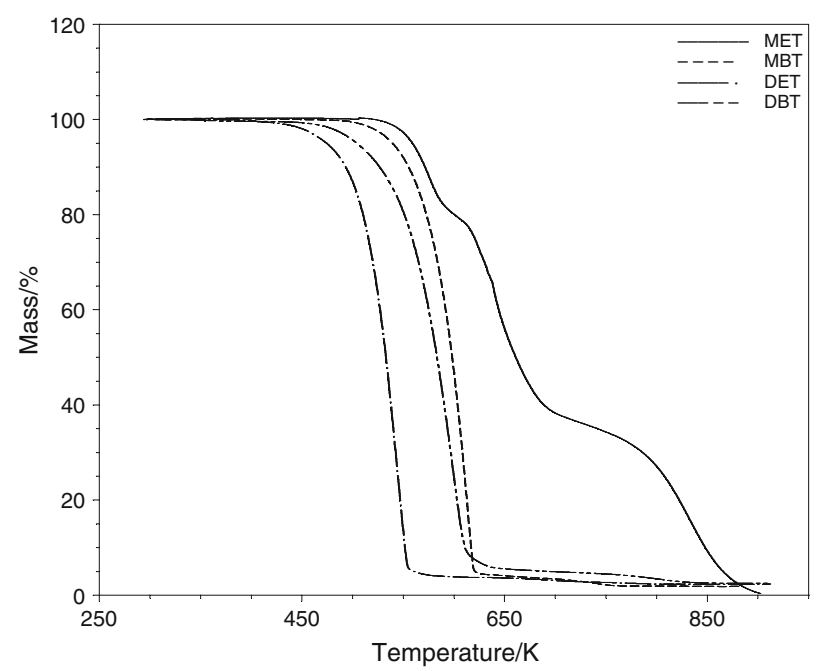

Fig. 2 Curve of terephthaloil-base amide modifiers

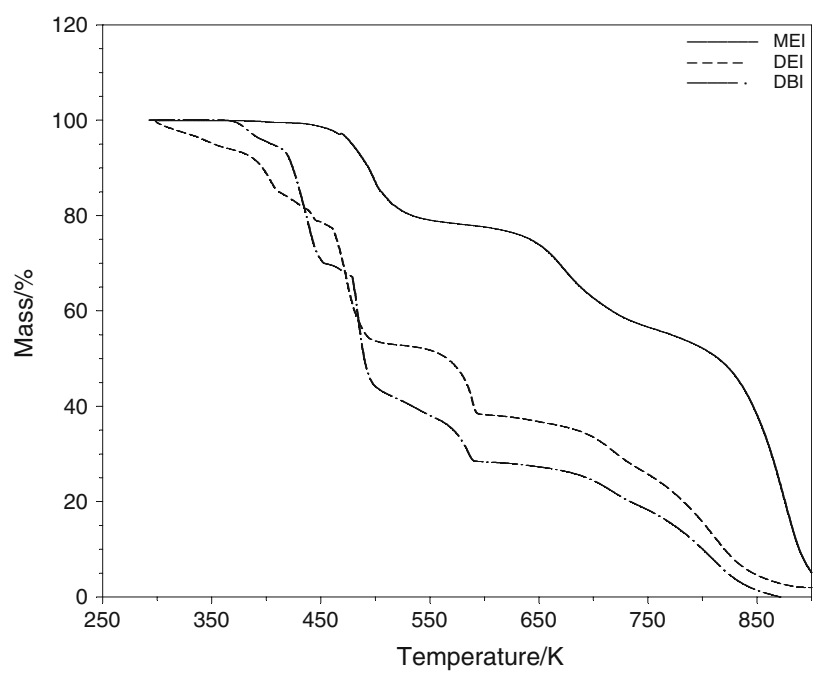

Fig. 3 Curve of 5-aminoisophthaloil-base amide modifiers

the analyses simulates real processing processes in conditions which require uncomplicated technology. It is clear that the obtained compounds exhibit thermal stability in the range 
of processing temperatures of many polymers. It can be observed that amides with single alkyl chain (MET and MBT) display greater thermal resistance, while amides DET and DBT undergo decomposition in lower temperatures. In the case of 5-aminoisophthalamides, the thermograms of DEI and DBI contained traces of solvent impurities while the greatest thermal stability of all the compounds was exhibited by $\mathrm{DBI}(478 \mathrm{~K})$, with relatively equal temperature in the case MEI and DEI of 463 and $461 \mathrm{~K}$, respectively.

Results of TG analysis of MMT modified with obtained amides are shown in Figs. 4 and 5 collective thermograms. Characteristic losses of mass at temperatures up to $400 \mathrm{~K}$ can be clearly seen. As concluded in previous researches, it is due to the water evaporation. In the case of clays with MET and MBT modifiers, water is linked stronger with those clays in comparison with the clays with DET and DBT modifiers. It is probably because the molecules of MET and MBT can exhibit the interaction with water molecules both with the free electron pair at nitrogen atom, and with the hydrogen atom bonded with this atom, which are not present in DET and DBT molecules. Moreover in Fig. 4, the thermogram of unmodified MMT is presented. That leads to a conclusion that applied modifiers do not have the negative influence on the thermal properties of basal clay. In comparison with thermogram shown in Fig. 3, lack of the significant changes, in the temperature range in which the modifiers decompose, can be observed. It proves the thermal stability of modifiers bonded with the clay. Thermogram 5 shows clearly that clays modified with amides DEI and DBI show greater stability than clay modified with MEI, which molecules undergo complicated decomposition stages and over $600 \mathrm{~K}$ temperature dramatic decomposition is observed.

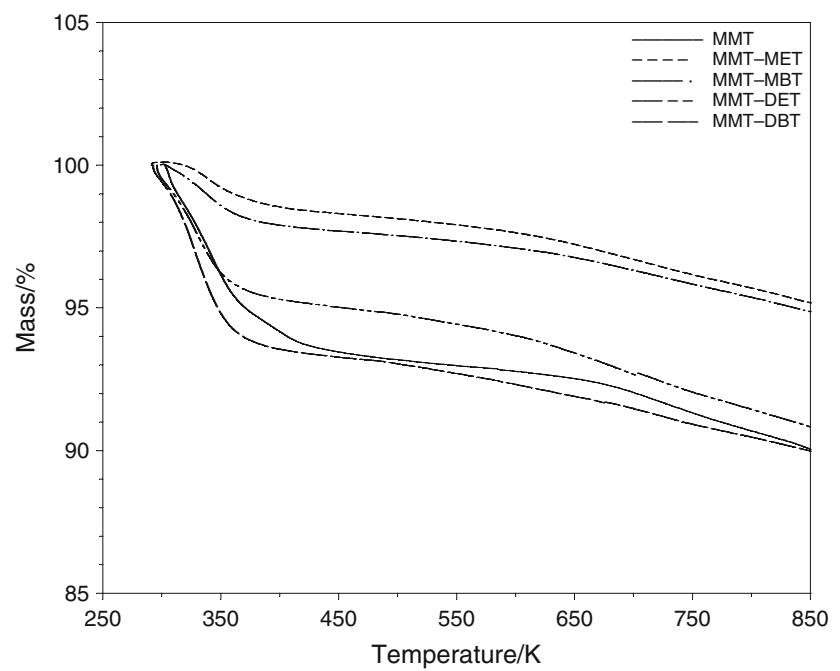

Fig. 4 Curve of unmodified and terephthaloil-base amides' modified clays

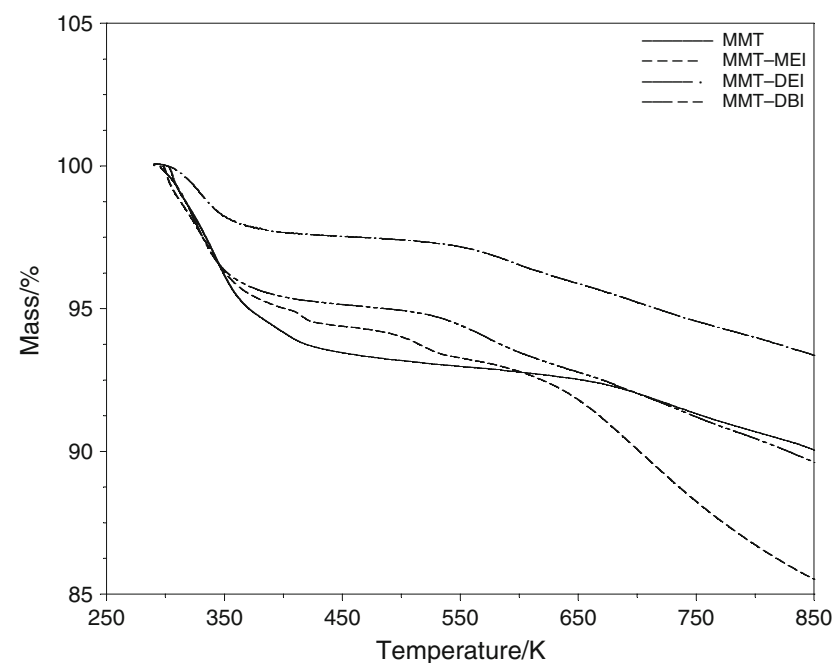

Fig. 5 Curve of unmodified and 5-aminoizophthaloil-base amides' modified clays

Figures 6 and 7 are worth mentioning. Figure 6 depicts thermograms of selected modifiers (DET and DBT) and clays modified with them (MMT-DET and MMT-DBT). It can be observed that incorporation of modifier into clay structure has got a positive influence on the thermal stability of modifier molecules. There is an effect of interaction of clay and modifier, which is seen in modified clay thermal decomposition processes in temperatures higher than temperatures of thermal decomposition of pure modifiers. The thermal stability effect is evident. Figure 7 exhibits comparison of selected modified clay, obtained directly from modification processes (MMT-DEI) and clay taken from the same batch, but temperature-treated in two cycles (MMT-DEI-reheated). Cycle consisted of heating

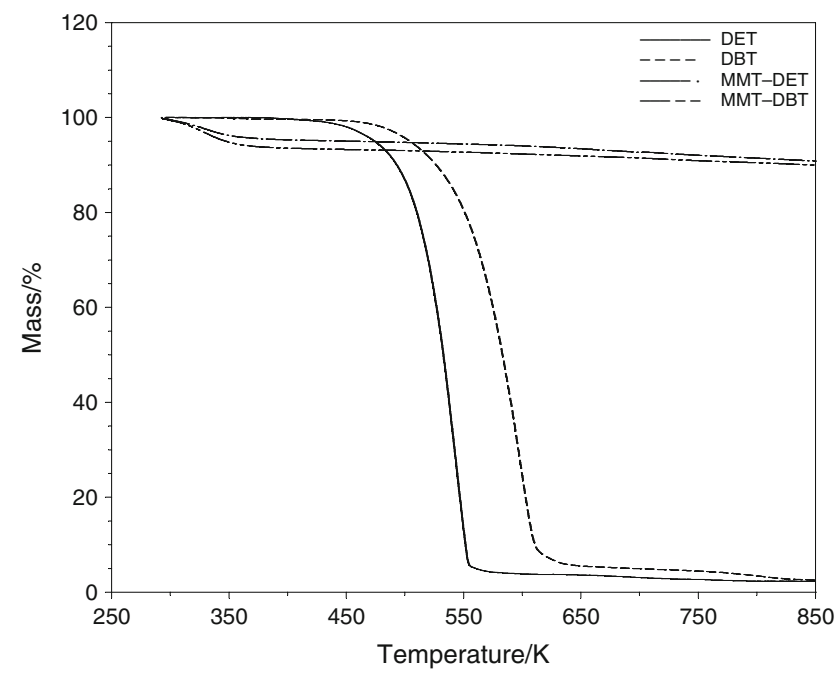

Fig. 6 Thermal stability effect of modified clay 


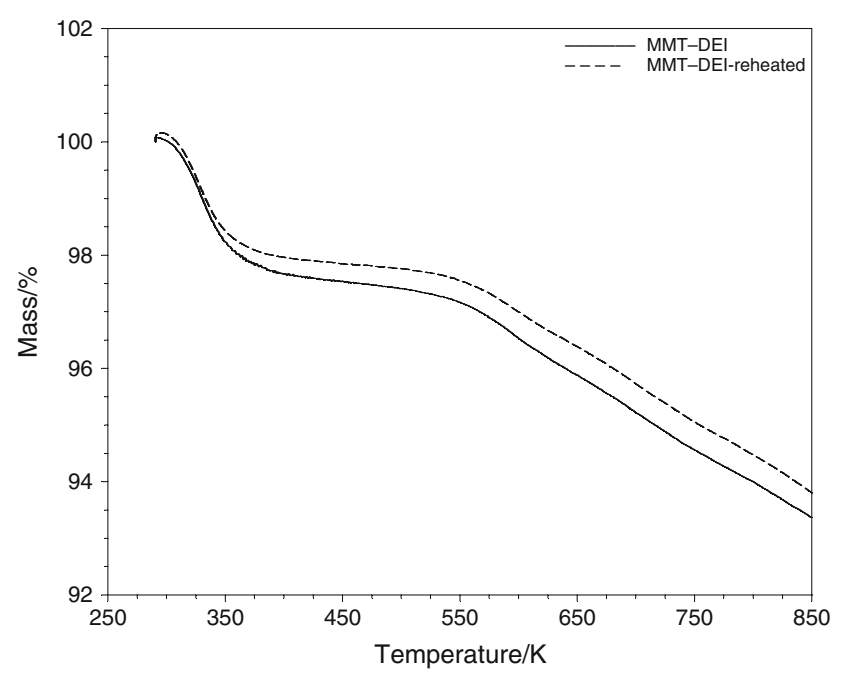

Fig. 7 The compare of modified and reheated modified clays

the sample up to the temperature of $473 \mathrm{~K}$ and keeping it in that temperature for $1 \mathrm{~h}$. That procedure was to simulate the processing methods with several heating times or to simulate the recycling of polymer nanocomposites with such clay used as a filler. As shown in Fig. 7 clay thermograms do not exhibit any changes in their run. That definitely confirms the possibility of multicycle processing of materials containing clays modified with investigated amide modifiers, with no negative influence on modifiers' thermal stability and modification effectiveness.

\section{NMR}

The results of all conducted synthesis were verified with NMR analysis to confirm the formation of proper products. ${ }^{1} \mathrm{H}$ NMR and ${ }^{13} \mathrm{C}$ NMR analyses were carried out with $\mathrm{CDCl}_{3}(\delta=7.26 \mathrm{ppm})$ and DMSO- $d_{6}(\delta=2.49 \mathrm{ppm})$ as solvents. At the ${ }^{1} \mathrm{H}$ NMR spectras of all synthesized compounds, the signals of methyl and methen groups of alkyl chains in range of 0.98-3.44 ppm (with amide characteristic effect of stopped alkyl chain rotation) are clearly visible. The signals of spectrally identical hydrogen atoms of terephthaloil and 5-amino-isophthaloli ring were observed at 7.30 and $7.66 \mathrm{ppm}$, respectively. The ${ }^{13} \mathrm{C}$ NMR analysis with additional usage of DEPT-135 method allowed to confirm carried out synthesis.

\section{XRD}

Modification processes were determined with XRD analysis to describe the changes of clays' intergallery distance. The XRD analysis justifies conclusions about the degree of clays' modification. The characteristic MMT reflection placed at $6.23^{\circ} 2 \theta$ comes from the ray's reflection on the (001) plane. In unmodified MMT that corresponds with the intergallery distance $d(001)$ of $0.96 \mathrm{~nm}$ [13]. The signal movement towards the smaller angle proves the increase of $d(001)$ distance, which indicates a more exfoliated structure. The complete lack of this signal means that the clays' structure is completely exfoliated. In polymer matrix such structure is so called unorganized exfoliated structure [14]. If the intergallery distance decreases, the signal moves towards the bigger angles at the roentgenogram. It may happen because of structural dehydration or the collapse of the structure in final polymer composite. A less intense and wider reflex (001) implies an intercalated-exfoliated structure [15]. Figure 8 presents the XRD patterns of unmodified MMT (a) and modified clays MMT-MET (b), MMT-MBT (c), MMT-DET (d), MMT-DBT (e), MMT-MEI (f), MMTDEI (g), MMT-DBI (h). It is clear that the characteristic signal of unmodified clay (a) moves towards the smaller angles to the complete disappearance in the case of (b)-(f) clays. That is the proof of their complete exfoliation, so the aim of modification processes is reached. Clays $(\mathrm{g})$ and $(\mathrm{h})$ present the (001) reflection at angles $6.68^{\circ} 2 \theta$ and $6.40^{\circ} 2 \theta$, respectively, which corresponds to intergallery distances 1.32 and $1.38 \mathrm{~nm}$, respectively. The decrease and widening of the
Fig. 8 XRD patterns of unmodified $(a)$ and modified $(b-h) \mathrm{MMT}$

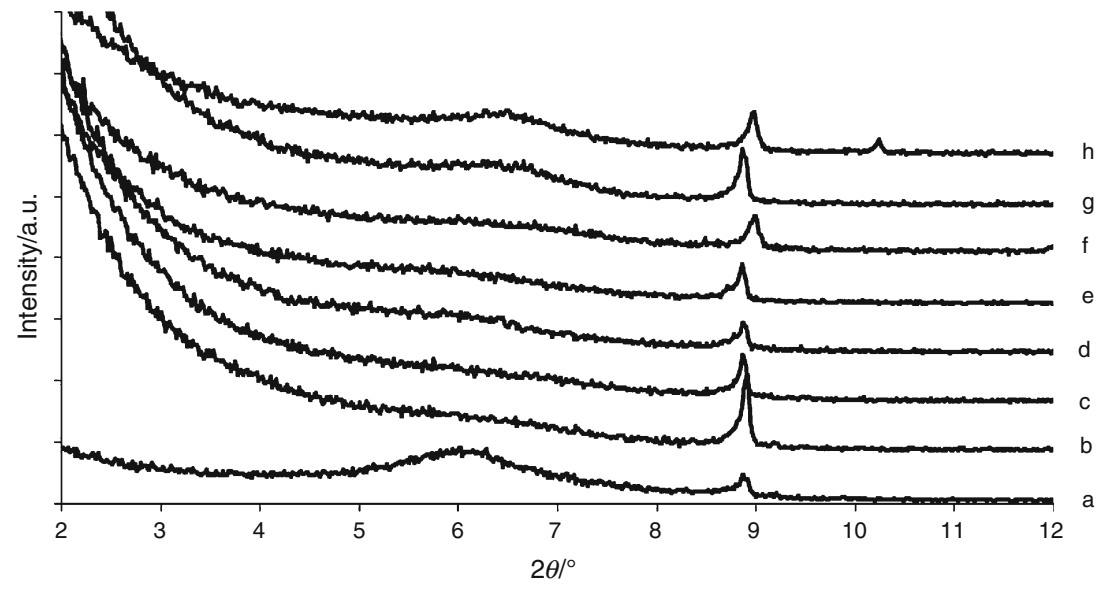


Table 2 Results of elementary analysis

\begin{tabular}{|c|c|c|c|c|c|c|c|c|}
\hline \multirow[t]{2}{*}{ Parameter } & \multirow[t]{2}{*}{ MMT } & \multicolumn{7}{|c|}{ MMT modified by } \\
\hline & & MET & MBT & DET & DBT & MEI & DEI & DBI \\
\hline$\% \mathrm{C}$ in sample/ \% & 0.063 & 0.69 & 0.52 & 0.89 & 0.21 & 1.37 & 1.29 & 4.68 \\
\hline Mass of modifier per $1{\mathrm{~g} \mathrm{MMT} / \mathrm{mg} \mathrm{g}^{-1}}^{-1}$ & - & 11.13 & 6.62 & 11.96 & 1.45 & 24.80 & 18.88 & 70.04 \\
\hline Quantity of modifier per $1 \mathrm{~g} \mathrm{MMT} / \mathrm{mmol} \mathrm{g}^{-1}$ & - & 0.04 & 0.02 & 0.04 & 0.01 & 0.09 & 0.06 & 0.17 \\
\hline$\%$ consumption of modifier/ $\%$ & - & 2.21 & 1.20 & 2.16 & 0.25 & 4.64 & 3.24 & 8.68 \\
\hline
\end{tabular}

signal intensity show that the clays' structure was partially exfoliated and the rest of it was deprived of intergallery water and modifier molecules covering the outer side of clay clusters. It can be caused by the specific structure of the DEI and DBI molecules, where the steric hindrance is big enough, so that the molecules do not incorporate into the intergallery space and the amine group influences the majority of the compounds chemisorption at the clay surface.

\section{Elementary analysis}

The elementary analysis was applied to determine the amount of modifiers effectively used in modification processes. The elementary analysis allows specifying the amount of, e.g., carbon in modified clays' samples (denote as: \% $\mathrm{C}$ in sample [\%]). Smectic clays do not have carbon atoms in their crystalline structure. This method allows detection of carbon coming only from modifier molecules. It can be reached by subtraction of the quantity of carbon in the unmodified MMT (as a result of natural sorption of carbon-containing compounds, e.g., $\mathrm{CO}_{2}$ ) from total $\% \mathrm{C}$ in sample. As shown in Table 2, after few calculations, it is possible to determine the amount of the modifier effectively combined with the clay. It is also possible to calculate the consumption of the modifier in the modification process, i.e., determining the relation between the amounts of modifier introduced into the modification system and the modifier combined with clay surface as the result of that process.

The obtained results allow a claim that the modification process was carried out. Because of different molecules structure, their conformation and interaction with clay's surface, it is difficult to describe the correlation between the amounts of the compound effectively modifying the clay in function of modifier structure and dimensions. The determined parameter of modificators usage enables to describe postprocessing amounts of modifying agent. From the technological point of view, it allows determining the effective usage and recovery of the modification process ingredients.

\section{Conclusions}

- The synthesis of new aromatic-aliphatic smectic clays' modifiers dedicated for PET and other polymers with aromatic parts in their structure was conducted.

- The research proved the effectiveness of modification of the tested compound of MMT clay.

- The XRD analysis confirmed the complete exfoliation of clays modified with MET, MBT, DET, DBT, and MEI compounds.

- Clays modified with DEI and DBI modifiers show a partially exfoliated structure with definitely dehydrated parts of clays' galleries.

- Presented molecules can be applied in polymer processing as smectic clay modifying agents in polyolefin and several other polymers.

- Modifier MET can be applied in PET processing with extrusion methods.

- MMT structure with the amide modifier exhibits thermal stability of the modifier's molecules.

- Modifiers' molecules showed the possibility of usage in the recycling cycles, on condition that the decomposition temperatures have not been exceeded.

- Molecules of MET amide exhibit the greatest thermal resistance of all presented modifying agents, but usage of each modifier depends on its polymer affinity and the polymer processing method used, e.g., to obtain polymer nanocomposites.

Acknowledgements The financial support from (1) the Faculty of Chemistry's Grants for young scientist No. 10/2011 and 24/2011 and (2) European Social Found, Polish National Budget within Integrate Operation Program of Regional Development, Action 2.6 „Regionalne Strategie Innowacyjne i transfer wiedzy” Kujawsko-pomorskie Voivodship home program „Stypendia dla doktorantów 2008/2009 ZPORR” are gratefully acknowledged.

Open Access This article is distributed under the terms of the Creative Commons Attribution License which permits any use, distribution, and reproduction in any medium, provided the original author(s) and the source are credited. 


\section{References}

1. Kozak M, Domka L. Adsorption of the quaternary ammonium salts on montmorillonite. J Phys Chem Solids. 2004;65:441-5.

2. Ding C, Jia D, He H, Guo B, Hong H. How organo-montmorillonite truly affects the structure and properties of polypropylene. Polym Test. 2005;24:94-100.

3. Lee SR, Park HM, Lim H, Kang T, Li X, Cho WJ, Ha CS. Microstructure, tensile properties, and biodegradability of aliphatic polyester/clay nanocomposites. Polymer. 2002;43: 2495-500.

4. Huang JC, Zhu ZK, Yin J, Qian XF, Sun YY. Poly(etherimide)/ montmorillonite nanocomposites prepared by melt intercalation: morphology, solvent resistance properties and thermal properties. Polymer. 2001;42:873-7.

5. Dang Z, Fan L, Shen Y, Nan C, Zhao S. Study of thermal and dielectric behavior of low-density polyethylene composites reinforced with zinc oxide whisker. J Therm Anal Calorim. 2012;71:635-41.

6. Morawiec J, Pawlak A, Slouf M, Gałeski A, Piórkowska E, Krasnikowa N. Preparation and properties of compatibilized LDPE/organo-modified montmorillonite nanocomposites. Eur Polym J. 2005;41:1115-22.

7. Rytwo G, Ruiz-Hitzky E. Enthalpies of adsorption of methylene blue and crystal violet to montmorillonite. J Therm Anal Calorim. 2012;71:751-9.
8. Solomons TWG. Organic chemistry. 4th ed. New York: Wiley; 1996.

9. Mitchell JA, Reid EE. The preparation of aliphatic amides. J Am Chem Soc. 1931;53:1879-83.

10. Ruhoff JR, Reid EE. A series of aliphatic dimethyl amides. J Am Chem Soc. 1937;59:401-2.

11. Roe ET, Scanlan JT, Swern D. Fatty acid amides. I. Preparation of amides of oleic and the 9,10-dihydroxystearic acids. J Am Chem Soc. 1949;71:2215-8.

12. Olewnik E, Garman K, Czerwiński W. Thermal properties of new composites based on nanoclay, polyethylene and polypropylene. J Therm Anal Calorim. 2010;101:323-9.

13. Bolewski A. Mineralogia szczegółowa. 3rd ed. Warszawa: Wydawnictwa Geologiczne; 1982.

14. Patino-Soto AP, Sanchez-Valdes S, Ramos-Devalle LF. Morphological and thermal properties of ABS/montmorillonite nanocomposites using two different ABS polymers and four different montmorillonite clays. J Polym Sci B. 2008;46: 190-200.

15. Palza H, Vergara R, Yazdani-Pedram M, Quijada R. Polypropylene/clay nanocomposites: effect of different clays and compatibilizers on their morphology. J Appl Polym Sci. 2009;112: $1278-86$. 